\title{
Elucidating reasons for failure to administer prescribed volume of nasogastric enteral feeds in a district general hospital
}

\author{
N. Bergin ${ }^{1}$, C. Kinder ${ }^{2}$ and A. Holdoway ${ }^{1}$ \\ ${ }^{1}$ Department of Nutrition \& Dietetics and ${ }^{2}$ Audit Department, Royal United Hospital, Combe Park, Bath BAl 3NG, UK
}

A number of studies have highlighted that the amount of enteral feed delivered is significantly less than that prescribed. The majority of these studies have been undertaken in the intensive care setting ${ }^{(1,2)}$. It has been demonstrated that patients on gastrostomy feeding were more likely to receive the prescribed feed when compared with nasogastric feeding (NGF) ${ }^{(3)}$. In hospital patients NGF is often the first route of enteral tube feeding. Amongst medical patients limited data are available to elucidate the reasons for failure of patients on NGF to receive the prescribed amount of feed. The aim of the present baseline audit was to establish the amount of feed administered $v$. that prescribed and identify reasons why patients failed to receive the volume of feed prescribed.

Data were collected prospectively on twenty consecutive patients who were commenced on NGF. Feed requirements were determined by a clinical dietitian. The volume of feed administered $v$. that prescribed was calculated from the daily fluid-balance charts. Method of feeding (pump $v$. bolus), medications and reasons for failure to administer prescribed feed were ascertained from medical, nursing and dietetic records and by questioning staff.

Data were evaluated for thirteen male and seven female patients (mean age 66 (range 35-91) years) on gastroenterology, oncology and ear, nose and throat wards. In total $235 \mathrm{~d}$ of NGF were prescribed and analysed. Mean NGF delivered was 77 (SD 39 ) \% of feed prescribed. Under-delivery of feed occurred on 70 of $235 \mathrm{~d}(30 \%)$. No feed was administered on 39 of $235 \mathrm{~d}$ (17\% of time period). Main reasons for not administering feed or administering less than that prescribed are illustrated in the Figure. The volume and energy deficit on days when NGF was less than that prescribed are detailed in the Table.

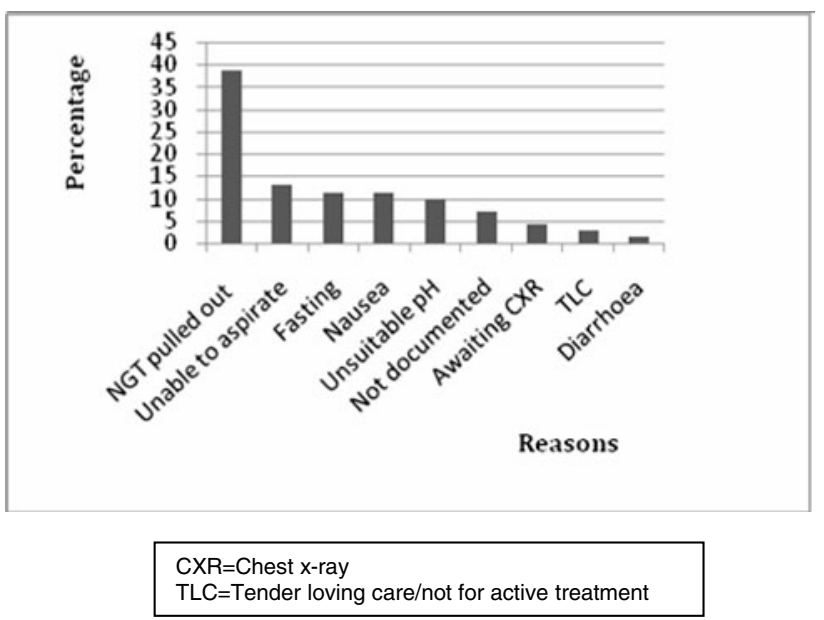

\begin{tabular}{ccc} 
Table. Volume $(\mathrm{ml} ; \%)$ and energy deficit (MJ; \%) for days when feed delivered \\
& Mean & SD \\
\hline & 669 & 339 \\
Volume deficit: $\mathrm{ml}$ & 83 & 59 \\
$\%$ & 3.15 & 1.59 \\
Energy deficit: MJ & 77 & 30 \\
$\%$ &
\end{tabular}

Figure. Reasons for failure to administer prescribed feed ( $\%$ total; $n 70)$. NGT, nasogastric tube.

In the current audit nasogastric tube (NGT) removal was the most common reason for patients not receiving the feed prescribed; patients with alcohol-induced liver disease accounted for $66 \%$ of these, a group who by their nature are restless. Difficulties confirming the correct tube position accounted for $27 \%$ of the days of under-delivery and $42 \%$ of the days on which no feed was administered. A total of $55 \%$ of patients were on proton pump inhibitors (PPI), which are known to increase gut $\mathrm{pH}$; this factor warrants further investigation as a possible cause of obtaining an unsuitable $\mathrm{pH}$ on aspiration.

Whilst some of the reasons for under-delivery of feed may be difficult to overcome, this initial audit highlights opportunities for improving practice to ensure that patients receive the prescribed feed. Inadvertent removal and inability to confirm correct positioning of NGT accounted for significant interruptions to feeding, resulting in under-delivery of feed and lost feeding days. As a result of this audit the nutrition support team are reviewing standards of care and procedures to improve methods of securing NGT, investigating aspiration practice, encouraging review of the need for PPI and optimising radiography procedures. The impact will be evaluated in due course.

1. Petros S \& Engelmann L (2006) Clin Nutr 25, 51-59.

2. Adam S \& Batson S (1997) Intensive Care Med 23, 246-247.

3. Park RHR, McAllison JL, Spence E et al. (1992) Br Med J 304, 1406-1409. 